\title{
SELF-SUSTAINED OSCILLATIONS IN A HETEROGENEOUS CATALYTIC REACTION: A MONTE CARLO SIMULATION
}

\author{
KRISIEN FICHTHORN, ERDOGAN GULARI and ROBERT ZIFF \\ Department of Chemical Engineering, University of Michigan, H. H. Dow Building, Ann Arbor, MI 48109, \\ U.S.A.
}

(Received 16 November 1987; accepted 13 June 1988)

\begin{abstract}
We have utilized Monte Carlo methods to study the kinetics of a generic heterogeneous catalytic reaction, $A+B \rightarrow A B$. This reaction includes the elementary steps of adsorption and desorption of reactants $A$ and $B$, surface reaction through the Langmuir-Hinshelwood mechanism, and desorption of product $A B$. It is shown that this model is capable of producing self-sustained oscillations in the rate of reaction. The oscillations are dependent on the rate of desorption and exhibit a time scale much greater than those of the adsorption and surface reaction steps in the model. We analyze the dynamic quality of the oscillations and discern that they stem from chaos. To our best knowledge, this is the first study in which chaos has been observed and characterized through a Monte Carlo simulation. With the results of this work, we have been able to analyze the fundamental components responsible for producing the chaos in our simulations. We discuss the implications of our results for actual catalytic systems with oscillatory behavior.
\end{abstract}

\section{INTRODUCTION}

In the recent literature, there has been considerable interest in catalytic systems with self-sustained oscillations in the rate of reaction. Oscillations have been found in several systems, the most notable of which is the $\mathrm{CO}$ oxidation reaction on platinum. The academic interest in such exotic phenomena is perhaps apparent from the voluminous theoretical and experimental literature on the oscillations (Razon and Schmitz, 1986; Sheintuch and Schmitz, 1977). Numerous mechanisms have been proposed to explain this behavior: however, at present, no single mechanism has been accepted unequivocally. At present, oscillations are generally attributed to a surface mechanism involving adsorbate-adsorbate and/or adsorbate-metal interactions. Several such mechanisms have been proposed and include: a dependence of the heat of adsorption on the coverage (Pikios and Luss, 1977; Rathausky and Hlavacek, 1981; Takoudis et al., 1981; Ivanov et al., 1980), non-uniformity of the catalyst surface (Prasad and Kulkarni, 1982; Jensen and Wray, 1980; Sault and Masel, 1982), a slow reversible oxidation of the catalyst metal (Eigenberger, 1978; Sales et al., 1982; Lindstrom and Tsotsis, 1985), and an adsorbateinduced phase transition of the catalyst surface-atoms [for example, the $1 \times 1 \Leftrightarrow$ hex transition of $\mathrm{Pt}(100)$ ] (Imbihl et al., 1985; Cox et al., 1985; Schwartz and Schmidt, 1987). All of these mechanisms have been proved to be theoretically and experimentally feasible and indicate that surface interactions of adsorbed molecules can contribute significantly to reaction kinetics and catalytic activity. Thus, it may become necessary to incorporate the effects of these surface phenomena in kinetic models to provide an accurate appraisal of reactor performance under prescribed operating conditions. With this incentive, it is useful to study surface phenomena and their effects on reaction kinetics. In a previous publication (Fichthorn et al.,
1988), we have discussed the advantages of utilizing Monte Carlo methods and fractal scaling relationships to accomplish this purpose were discussed. We demonstrated the Monte Carlo method through simulation of a generic bimolecular LangmuirHinshelwood reaction

$$
A_{g}+B_{g} \underset{\text { surface }}{\stackrel{\text { Catalytic }}{\longrightarrow}} \mathbf{A} \mathbf{B}_{\mathrm{g}}
$$

which we have designated the AB model. Although the only surface mechanism in this model is the Langmuir-Hinshelwood reaction, we found that adsorbate islands were formed on surfaces in our simulations and we measured several of their identifying fractal attributes. We also discovered that the slow growth of these islands led to the eventual selfpoisoning of surfaces in our simulations. In this paper we show that the self-poisoning tendency of the irreversible model can contribute to self-sustained oscillations in the rate of reaction of simulations with reversibility. We observe that these oscillations are a cooperative surface phenomenon with a time scale much greater than those of the elementary steps (e.g. adsorption, surface reaction) in our simulation. Through dynamic analysis of our oscillatory rate of reaction time series, we conclude that they stem from deterministic chaos. The innate simplicity of our model allows us to isolate the elements responsible for the chaos and provide an interpretation of oscillatory behavior in actual catalytic systems with similar features.

\section{THE MODEL SYSTEM}

The model reaction proceeds through the mechanism

$$
\mathrm{A}_{\mathrm{g}}+\mathrm{S} \underset{k_{d}}{\stackrel{k_{a}}{\rightleftarrows}} \mathrm{A}-\mathrm{S}
$$




$$
\begin{gathered}
\mathbf{B}_{\mathrm{g}}+\mathrm{S} \underset{\mathbf{k}_{d}}{\stackrel{k_{\mathrm{a}}}{\rightleftharpoons}} \mathrm{B}-\mathrm{S} \\
\mathrm{A}-\mathrm{S}+\mathrm{B}-\mathrm{S} \stackrel{\boldsymbol{k}_{r}}{\longrightarrow} \mathrm{A} \mathrm{B}_{\mathrm{g}}+2 \mathrm{~S}
\end{gathered}
$$

where $k_{c}, k_{d}$ and $k_{r}$ are the rate constants for adsorption, desorption and surface reaction, respectively, and $S$ denotes a vacant site on the catalyst surface. This mechanism is not intended to represent that of any actual catalytic reaction: rather, we selected the model to characterize a generic, bimolecular LangmuirHinshelwood reaction. At present, we assume a uniform catalytic surface and our only surface mechanism is a Langmuir-Hinshelwood reaction. Despite the innate simplicity of this mechanism, our system exhibits a remarkable wealth of kinetic behavior when simulated with Monte Carlo methods. This kinetic behavior is influenced largely by the growth dynamics of adsorbate islands which form on the simulation surfaces. Wicke et al. (1980) first observed these islands in a Monte Carlo simulation of the irreversible $\left(k_{d}=0\right)$ surface reaction limited $\left(k_{a} \gg k_{r}\right)$ version of the AB model. Our group later confirmed that adsorbate islands were formed in the irreversible AB model regardless of the relative rate of adsorption to reaction (Fichthorn et al., 1988; Ziff and Fichthorn, 1986). In addition, we found that the slow growth of these islands leads to the eventual poisoning of the catalyst surface in systems without desorption. The time of this poisoning has a power-law dependence on the size of the surface in our simulation

$$
T_{\mathrm{p}} \sim L^{2}
$$

where $T_{p}$ is the poisoning time in Monte Carlo steps (to be defined subsequently), $L$ is the linear dimension of our square surfaces, and $z$ is the poisoning exponent with a value of $2.1 \pm 0.03$ for all kinetic regimes. From the results of the irreversible model, it is determined that the necessary elements for island formation and growth are the Langmuir-Hinshelwood mechanism and, to some extent, the irreversibility of reactant adsorption. In this paper, we quantify the extent to which irreversibility of reactant adsorption influences the poisoning of surfaces by introducing the parameter of the rate of spontaneous reactant desorption to our simulations. We will show that, through manipulation of this parameter, we can achieve catalytic activity ranging from stationary to oscillatory to poisoning.

\section{THE MONTE CARLO ALGORITHM}

The algorithm for Monte Carlo simulation of our model reaction is depicted in Fig. 1. We utilize a twodimensional computer array to represent the catalyst surface. The surfaces in our simulations are uniform with square coordination and periodic boundary conditions. Initially, we populate entire catalytic surface randomly and homogeneously with reactants $A$ and $B$. We begin the simulation by picking a site on the catalyst surface at random. We then choose desorption with probability $P_{d}$, or surface reaction with

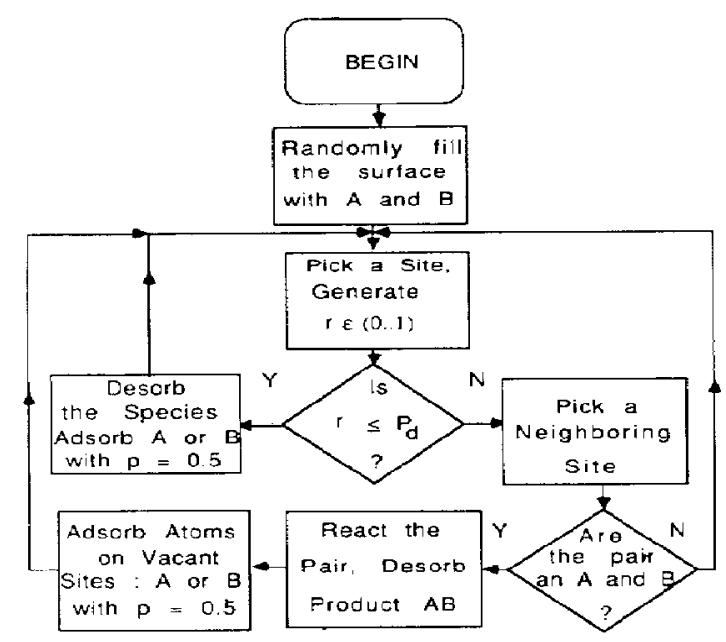

Fig. 1. Flow diagram of the model reaction algorithm. The catalyst surface is represented by a two-dimensional computer array with square coordination and periodic boundary conditions.

probability $P_{r}\left(=1-P_{d}\right)$. If desorption is selected, we remove the reactant from its site on the catalyst surface and immediately adsorb another in its place (A or $B$ with equal probability). If surface reaction is chosen, we pick a neighboring site at random (one of four for square coordination) and remove the molecule and its neighbor if the pair are an $A$ and $B$. Two adsorptions, where $A$ and $B$ are chosen to adsorb with equal probability, immediately succeed each reaction. This algorithm is run repeatedly, into the long-time regime, while we monitor the rate of reaction and surface coverage of reactant as a function of time. Time in Monte Carlo simulations is measured in Monte Carlo steps (MCS). We define 1 MCS as $N$ trials (starting from "pick a site") from the algorithm of Fig. 1, where $N$ is the total number of sites on our catalytic surface. An MCS is directly proportional to real time in an actual catalytic reaction. Given the parameters (e.g. pressure, temperature, activation energies, sticking coefficients) for a catalytic reaction, we can calculate rate constants and, thus obtain time scales for each step in the simulation.

The algorithm of Fig. 1 has been designed to accommodate several specific parameters from the system of eqs (2)-(4). We assume the gas phase partial pressures of reactants $A$ and $B$ are equal and we also set the rate constants for adsorption and desorption of $A$ equal to those for B. These stipulations were chosen to satisfy the stoichiometry of the reaction so that maximal rates of reaction could be achieved. The algorithm of Fig. 1 reflects this convention through the rule that $A$ and $B$ are chosen for adsorption and desorption with equal probability.

Our Monte Carlo algorithm is also designed to simulate surface reaction limiting kinetics $\left(k_{a} \gg k_{r}\right)$. Since we had previously ascertained that the qualitative kinetic behavior in our simulations was insensitive 
to the relative rate of adsorption to surface reaction, we could assign this ratio somewhat arbitrarily. We choose surface reaction limiting kinetics for computational convenience. In the surface reaction limiting regime, adsorption occurs rapidly compared to reaction. Thus, in the time scale of a reaction, we expect adsorption to occur on every available site. As soon as a site is vacated, adsorption immediately follows. This convention is implicit in the algorithm of Fig. 1 through the initial condition of a full surface and the rule that one or two adsorptions must succeed each desorption or surface reaction, respectively.

The desorption probability, $P_{d}$, of Fig. 1 is the adjustable parameter in our simulations. With adsorption as the fast-step, the desorption probability defines the relative rate of desorption to surface reaction:

$$
P_{d}=\frac{k_{d}}{k_{r}+k_{d}} \text {. }
$$

We found that the kinetic behavior of our simulations was extremely sensitive to this parameter. Thus, we ran simulations over an encompassing range of desorption rates $\left(k_{r}>k_{d}>0\right.$, or $\left.P_{d} \leq 1 / 2\right)$ to study this dependence.

An additional parameter in our simulations is the size of our system. To obtain valid conclusions from Monte Carlo simulations, it is essential to determine the dependence of results on this parameter. We ran most of our simulations on $32 \times 32$ surfaces $\left(2^{10}\right.$ sites $)$ : however, we also obtained results from $16 \times 16$ and 64 $\times 64$ surfaces. These sizes were chosen to facilitate our calculations and allowed us to run our simulations into the long-time regime (greater than $150,000 \mathrm{MCS}$ ). Thus, we could obtain time series of our results and the assurance that the initial relaxation time had been greatly exceeded.

Thus, the rate of desorption and the size of our surfaces were manipulated variables affecting the response of our system. We measured this response by monitoring the rate of reaction and the fractional surface coverage of $A$ as a function of time for all simulations. We define the rate of reaction as the number of reactions per site in 1 MCS and the fractional coverage of $A$ as the ratio of the number of surface sites occupied by $A$ to the total number of sites. We measured these values every $10 \mathrm{MCS}$ in all simulations. Also, with the graphics capabilities of the Apollo computer, we were able to watch our surfaces as the simulations ran and observe changes in their configuration with time.

\section{RESULTS}

Figure 2 shows the rate of reaction as a function of time as the rate of desorption is decreased over five orders of magnitude on a $32 \times 32$ surface. For relatively large rates of desorption [Fig. 2(a) and (b)], we see that the rates of reaction assume essentially stationary values. However, from Fig. 2(a) to Fig. 2(b), we notice an increasing noise level in the rate of reaction as the rate of desorption is decreased. In Fig. 2(c), this "noise level" has increased to the point where fluctuations in the rate become relatively large in magnitude. In Fig. 2(d), oscillations completely dominate the rate of reaction, and, finally, in Fig. 2(e), the rate of desorption is sufficiently small so that, after an initial transient, the lattice is essentially poisoned. Thus, by decreasing the rate of desorption in simulations, we obtain kinetic behavior ranging from

(a)

(b)
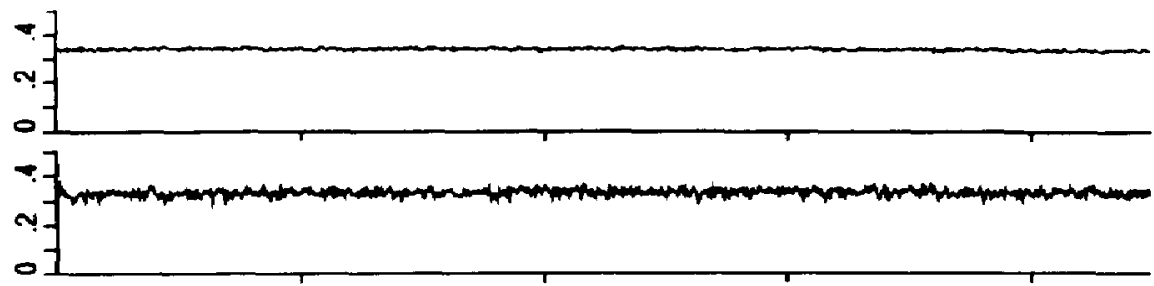

(c)

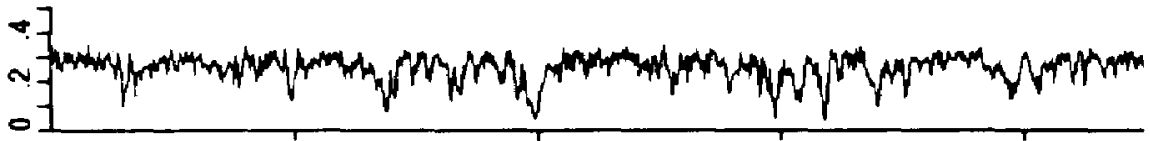

(d)

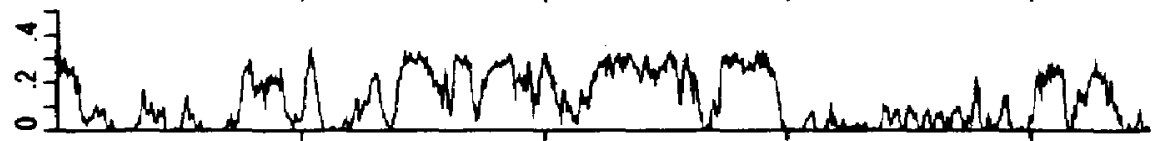

(e)
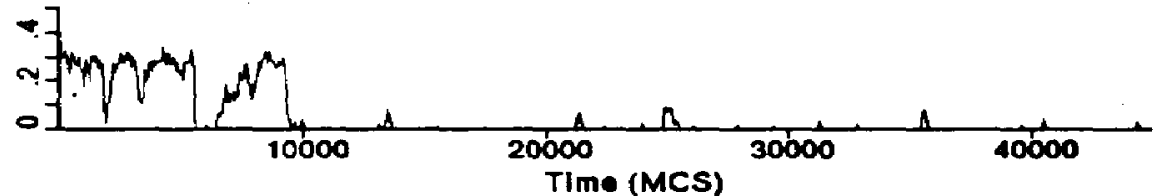

Fig. 2. Rate of reaction (reactions per site per MCS) as a function of time (MCS) for successively lower values of the desorption probability, $P_{d}$, on a $32 \times 32$ lattice: (a) $P_{d}=0.2$, (b) $P_{d}=0.02$, (c) $P_{d}=0.002$, (d) $P_{d}$ $=0.0002$, (e) $P_{d}=0.00002$. 
steady state to oscillatory to virtually poisoning.

The rate oscillations in our systems were synchronous with oscillations in surface coverage. Figure 3 shows the rate of reaction and the fractional coverage of $A$ as a function of time on a $32 \times 32$ lattice. From these plots, we see that minima in the rate of reaction correspond to coverages of zero and one which are configurations poisoned with $\mathbf{B}$ and $\mathbf{A}$, respectively. Maxima in the rate of reaction coincide with fractional coverages near 50\%. Figure 4 follows the rate and coverage plots of Fig. 3 and depicts reactant configurations on the surface of Fig. 3 at several points. When the surface is poisoned with a single species, say $\mathbf{A}$, the rate of reaction is zero, as is the case at $15,000 \mathrm{MCS}$ in Fig. 3(a). At this point, desorption occurs and allows one B to subsequently readsorb. This B reacts, clearing two active sites, and allows two more Bs to adsorb with $25 \%$ probability. This process continues until a region of $B$ emerges, as depicted in Fig. 4(a), taken at $15,500 \mathrm{MCS}$. Of course, in this process, there is a substantial probability that the surface will return to its original poisoned configuration. However, occasionally, the region of $\mathbf{B}$ evolves to become several small islands, as shown in Fig. 4(b) at 16,500 MCS. From this point, successive reactions and desorptions create a still larger region of $B$ [Fig. 4(c) at 17,500 MCS] which is eventually broken up as it is consumed by the increasing rate of reaction. This produces a roughly homogeneous distribution of $A$ and $B$ with a maximum reaction rate, as shown in Fig. 4(d) at 19,000 MCS. From this point, the islands begin to grow and coalesce and the rate of reaction decreases until poisoning with $\mathbf{A}$ or $\mathbf{B}$ occurs and the cycle repeats.

With a qualitative understanding of the oscillatory behavior in our simulations, we sought more quantitative measures. Specifically, we addressed the following questions:

(1) How are kinetics dependent on the rate of desorption for a given system size?

(2) How does the system size affect kinetic behavior?
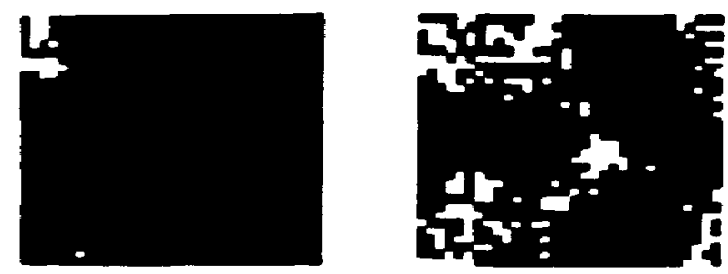

(a)

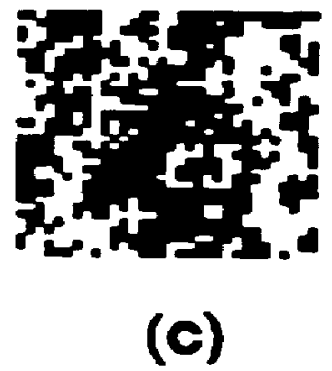

(b)

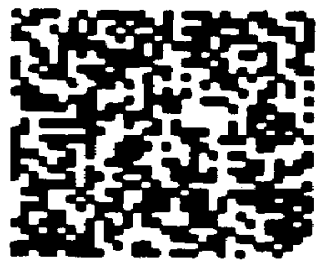

(d)
Fig. 4. Snapshots of the surface from a simulation on a 32 $\times 32$ lattice with $P_{d}=0.0001$. The black area is reactant $A$ and the white area is B. (a) $15,500 \mathrm{MCS}$ our surface has one small region of $B$ surrounded by $A$. (b) $16,500 \mathrm{MCS}$ - the region of $B$ has grown to several small islands. (c) 17,500 MCS-the $A$ and $B$ regions are approximately equivalent; however, A and B still exist in large islands. (d) 19,000 MCS-the islands have dispersed, leaving a homogeneous distribution of $A$ and $B$ on the surface.

and

(3) I Low do we classify the kinetic behavior dynamically?

The dependence of the kinetic behavior on the rate of desorption is depicted qualitatively in Fig. 2. From Fig. 2, we see that oscillations arise from the amplification of noise through decreasing desorption rates in

(a)

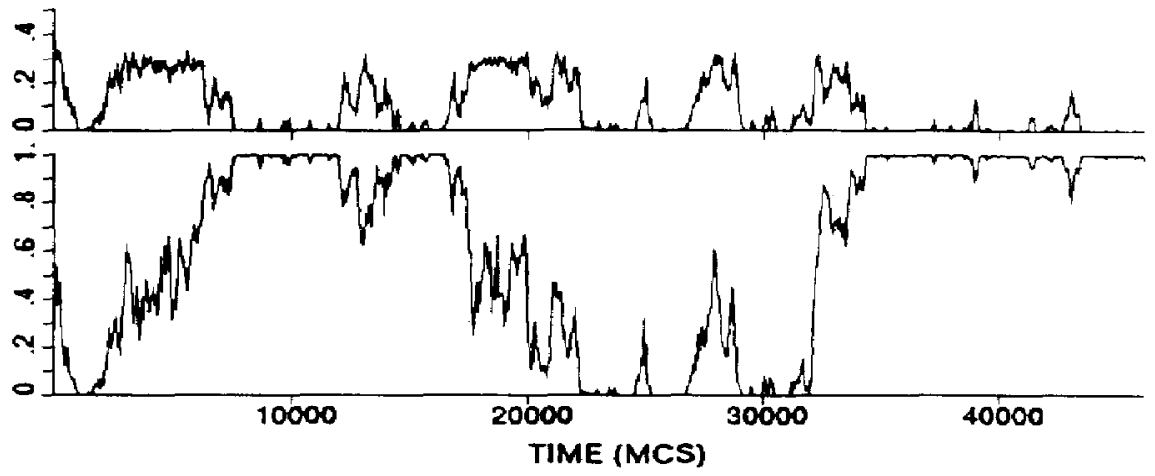

Fig. 3. Rate of reaction (a) and fractional surface coverage of $A(b)$ as a function of time for a $32 \times 32$ lattice with $P_{d}=0.0001$. 
our systems. For our first assessment of the oscillations, we mcasurcd this amplification as a function of the rate of desorption. We calculated the average rate and the mean-square deviation from the average, or the amplitude of the oscillations, for every time series obtained in our simulations. Figure 5 shows a semi-log plot of this measure for three lattice sizes. For a given lattice, the amplitude of the oscillations reaches a maximum. Through observation of our surfaces during simulation, we found that this maximum corresponds to the desorption rate for which the system first reaches a poisoned configuration. For desorption rates above this maximum, our system fluctuates between nonpoisoned configurations, and for rates decreasing below the maximum, the systems spend an increasing time in the poisoned configuration. We also see from Fig. 5 that the maximum of the oscillation amplitude decreases as the system size increases. This indicates that the oscillations are a finite size effect: we would not expect them in an infinite system.

Figure 6 shows the average rate of reaction as a function of the rate of desorption. From this plot, we see that maxima in the amplitude of the oscillations do not produce corresponding maxima in the average rate. We expect our rate of reaction to increase monotonically with the rate of desorption because desorption disperses islands, thereby making the surface more homogeneous and surface reaction more probable. The maximum in Fig. 6 occurs when the desorption rate is sufficiently large to be competitive with surface reaction. It appears that this maximum is independent of the system size, presumably because the reactant islands are small compared to the size of our surfaces at this point. As the rate of desorption decreases from the maximum, however, we see the effects of the system size on the average rate of reaction. Larger systems are less prone to fluctuations

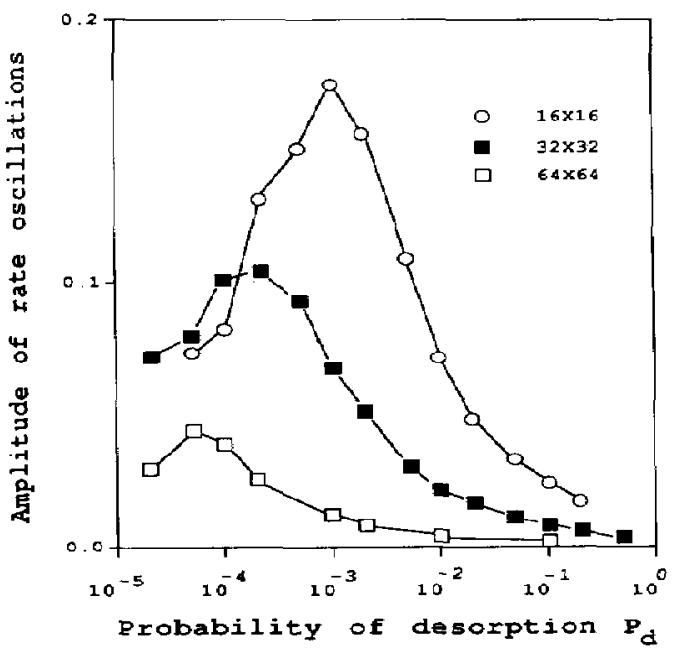

Fig. 5. Mean-square deviation of the rate of reaction as a function of the desorption probability for $a+16 \times 16,32 \times 32$ and $64 \times 64$ surface.

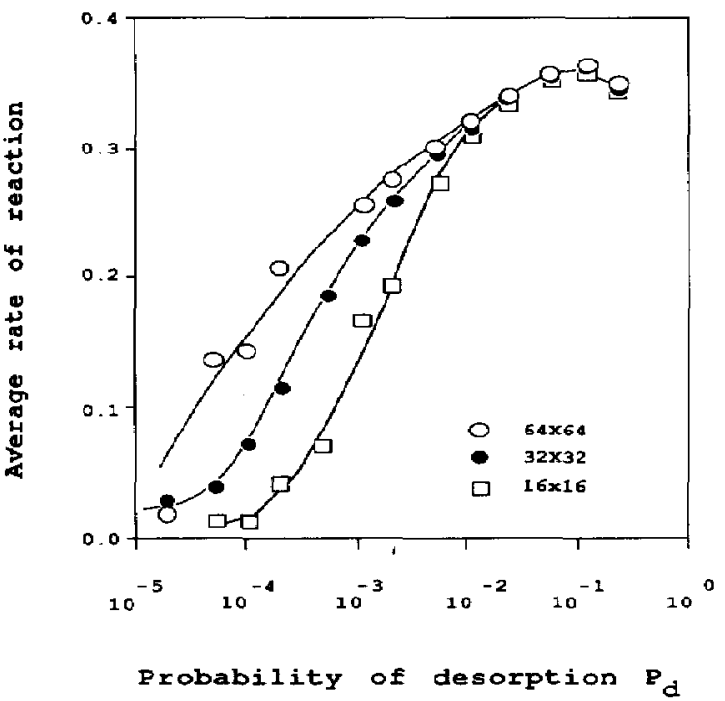

Fig. 6. Average rate of reaction as a function of the probability of desorption for $16 \times 16,32 \times 32$ and $64 \times 64$ lattices. The average consists of measurements made every $10 \mathrm{MCS}$ through the duration of the simulations $(50,000-500,000$ steps).

in the surface configuration and, thus, maintain higher average reaction rates.

To further define the quality of our rate and coverage oscillations, we analyzed our time series to ascertain their dynamic classification. For relatively high rates of desorption, our rate of reaction remained nearly stationary, as shown in Fig. 2(a) and (b). In a dynamical notation, we classify this steady state as a fixed point. As the rate of desorption in our simulation is decreased, the fixed point becomes blurred by increasing noise fluctuations in the system, as seen in Fig. 2(c). Finally, as the rate of desorption is further decreased, the amplitude of the fluctuations exceeds the average rate of reaction. From Figs 5 and 6 , we see that this occurs at the approximate rate of desorption where the fiuctuations reach a maximum amplitude. At this point, our basin of attraction is no longer a fixed point, or a single steady state, but a more complex attractor. Since our oscillations seemed highly aperiodic, or chaotic, at this point of maximum amplitude, we sought to characterize the "strange attractor" underlying our dynamics. Dynamical information can also be extracted from time series obtained experimentally and, thus, be matched with our findings.

We utilized the correlation integral method (Grassberger and Procaccia, 1983a, b) to analyze our rate of reaction time series. Given a time series

$$
\left\{\mathbf{x}_{i}, i=1, \ldots, N\right\}
$$

taken at times $i \tau$, where $\tau$ is the time interval between the $N$ successive measurements in the series, the correlation jntegral over a given length scale, $l$, is 
defined as

$$
\begin{aligned}
& C(l)=\lim _{\substack{N \rightarrow \infty \\
N}} 1 / N^{2}\{\text { the number of pairs } i, j \text { such } \\
& \text { that } \left.\left|\mathbf{x}_{i}-\mathbf{x}_{j}\right|<l\right\} .
\end{aligned}
$$

When the correlation integral is calculated over varying length scales, it obeys the scaling relationship

$$
C(l) \sim l^{v}
$$

for small length scales. In this relationship, $v$ is the correlation exponent. The correlation exponent characterizes spatial correlations between successive values in the time series and, thus, describes the homogeneity of the series in phase space. When the dimensionality of $x_{i}$ in eq. (7) is low, the series can be embedded in higher-dimensional space to eliminate error in the measurement of $v$. This embedding is accomplished by creating $d$-dimensional vectors from the time series:

$$
\xi_{i}=\left(\mathbf{x}_{i}, \mathbf{x}_{i+\tau}, \ldots, \mathbf{x}_{i+(d-1) \tau}\right)
$$

and substituting $\xi_{i}$ for $x_{i}$ in eq. (8). When we measure the correlation exponent from eqs $(8)-(10)$ as the series of eq. (7) is embedded in increasing dimensions, its value will increase with the embedding dimension if the series is random. If the series is governed by a strange attractor, the correlation exponent will assume a constant value, independent of the embedding dimension. For a chaotic series, the correlation exponent follows the relationship

$$
v \leq D_{f}
$$

where $D_{f}$ is the fractal dimension of the strange attractor. The fractal dimension is necessarily less than or equal to the dimensionality of the time series, $\mathbf{x}_{\boldsymbol{i}}$. For our system, the dimensions of our rate and coverage time series were both one and this method was employed to calculate $v$.

Figure 7 shows a plot from which we calculated the correlation exponent from the rate of reaction oscillations on a $32 \times 32$ lattice at $P_{d}=0.0001$, approximately the point where the oscillations reach a maximum in amplitude. This time series consisted of 10,000 points spaced $10 \mathrm{MCS}$ apart $(\tau=10)$. The correlation exponent, which is obtained from the slopes of successive lines, was measured to be $0.37 \pm 0.03$. Figure 8 shows a similar plot for the coverage time series corresponding to the rate series of Fig. 7 . This plot was obtained from a series of 25,000 points spaced $20 \mathrm{MCS}$ apart $(\tau=20)$. The correlation exponent for this series was $0.38 \pm 0.01$, matching our exponent for the rate series within the limits of error. The slopes for the higher dimensions (e.g. $D=3$ or 4) from both of these plots were calculated utilizing points at longer length scales. The points at the smaller length scales and higher dimensions of Figs 7 and 8 are affected by the noise level of our system. This phenomenon is a welldocumented fact in the literature (Ben-Mizrachi et al., 1984) and can be corrected by utilization of more data points in the analysis of the series. For our work, the

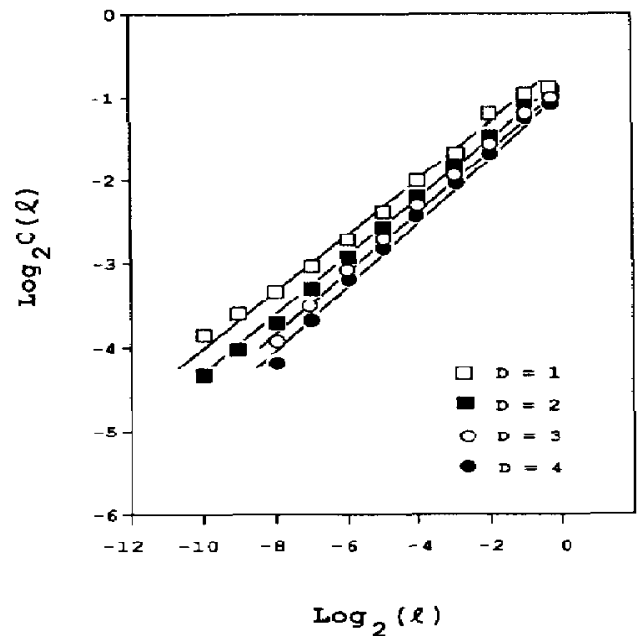

Fig. 7. Log log plot of the correlation integral [eq. (8)] for our rate of reaction time series over various length scales, $l$. The time series was obtained for a $32 \times 32$ lattice with $P_{d}$ $=0.0001$ and consists of 10,000 points measured at $10 \mathrm{MCS}$ intervals $(\tau=10)$. As we embed the time series in increasing dimensional space $(D=1, \ldots 4)$, the correlation exponent, which is the slope of successive lines, retains a constant value of $0.37 \pm 0.03$.

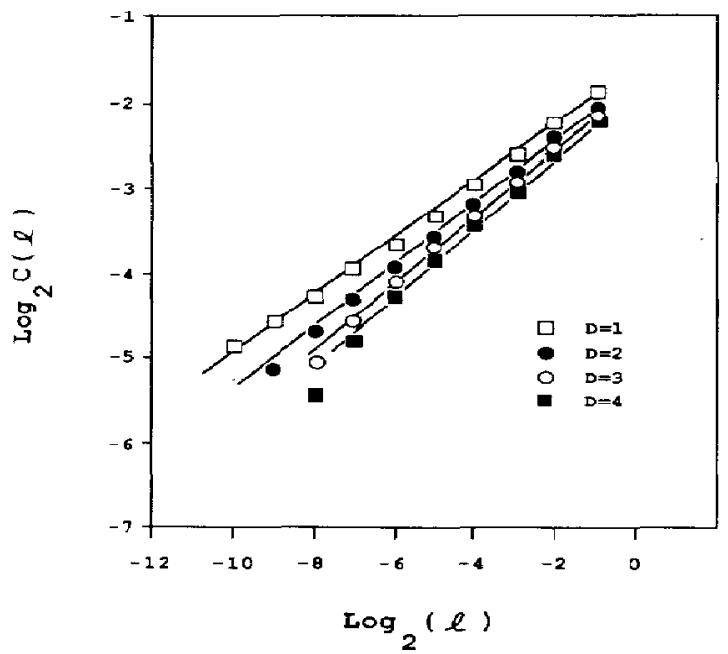

Fig. 8. Log-log.plot of the correlation integral [eq. (8)] for the coverage time series corresponding to the rate of reaction time series of Fig. 7. The series consists of 25,000 points measurcd at $20 \mathrm{MCS}$ intervals $(\tau=20)$. The correlation exponent for this time series is $0.38 \pm 0.01$.

computation time became prohibitively long for time series with more points.

Thus, with a Monte Carlo simulation, we have synthesized deterministic chaos. However, to ensure that this chaos was generated by our reaction algorithm and not our random number generator, we also measured the correlation exponent for our random number generator. The distinction between randomness and chaos is not always clear-cut and 


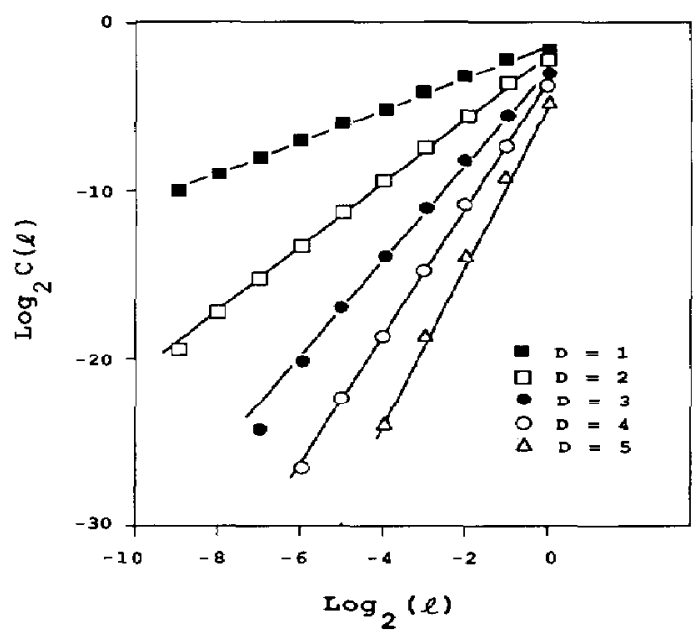

Fig. 9. Log-log plot of the correlation integral [eq. (8)] for a series of numbers from our random number generator. The series consists of 10,000 numbers.

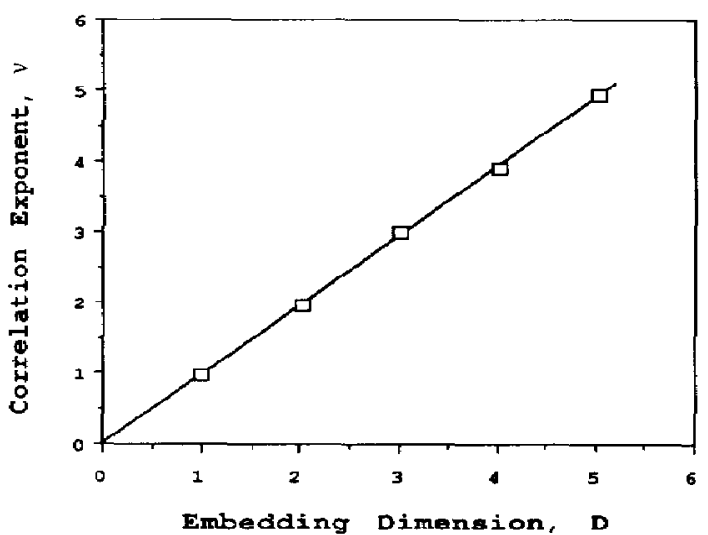

Fig. 10. Correlation exponent as a function of embedding dimension for our random number generator. The slope of the line, which is approximately 1.0 , indicates the randomness of the numbers.

complete randomness is generally not attainable with number-generating algorithms. However, we determined that our generator was random to the degree that we could detect, as shown in Figs 9 and 10. In Fig. 9, we calculated the correlation integral for varying length scales for a series of 10,000 random numbers from our number generator. Figure 10 shows a plot of the correlation exponent measured from Fig. 9 as a function of the embedding dimension. The slope of this line is one, indicating the randomness of the numbers.

\section{DISCUSSION AND CONCLUSIONS}

Thus, in a relatively simple model of a bimolecular catalytic reaction, we achieve a wealth of interesting and unanticipated dynamic behavior. For irreversible: systems, the island growth dynamics induced by the Langmuir-Hinshelwood mechanism leads to poisoning of the catalytic surface. We find that poisoning can be avoided in systems with reactant desorption and that steady-state, reactive surface configurations can be attained. For sufficiently high rates of desorption, we find that the rates of reaction measured from our simulations remain nearly stationary. However, as the rate of desorption is decreased from its stationary value, we find that the rate of reaction and surface coverage become more unstable and oscillate with increasing amplitude. The amplitude of the oscillations increases until the point where our systems reach the poisoned configuration. At this point, as the rate of desorption is decreased to zero, the amplitude of the oscillations also decreases and our systems spend increasing amounts of time in the poisoned configuration. We also find that the amplitude of our oscillations decreases with increasing system size. From this observation, we predict that we would not observe oscillations in a sufficiently large system. We analyzed the oscillatory dynamics of our rate of reaction and coverage time series using the correlation integral method. From this analysis, we concluded that the oscillations were chaotic with a correlation exponent $v=0.37$.

The apparent fact that we have generated chaos with a Monte Carlo simulation is somewhat surprising since, to our knowledge, this has not been achieved nor attempted previously. Thus, a major finding in this work is the identification of the potential of utilizing Monte Carlo simulations to study the origins of chaos. This result has applications in many branches of physics where nonlinear dynamics are inherent, including surface science. A more complete analysis of the dynamic behavior of this system is presented elsewhere (Fichthorn et al., 1989)

Earlier studies (Ziff et al., 1986) have indicated that Monte Carlo simulations may be useful in understanding oscillatory behavior in the $\mathrm{CO}$ oxidation reaction. In this work, an irreversible system was studied whose mechanism was chosen to represent the most widely-accepted mechanism for the $\mathrm{CO}$ oxidation reaction (Engel and Ertl, 1979):

$$
\begin{aligned}
& A_{\mathrm{g}}+\mathbf{S} \stackrel{\boldsymbol{k}_{\mathrm{a} 1}}{\longrightarrow} \mathrm{A}-\mathrm{S} \\
& \mathrm{B}_{2 \mathrm{~g}}+2 \mathrm{~S} \stackrel{k_{\mathrm{a} 2}}{\longrightarrow} 2 \mathrm{~B}-\mathrm{S} \\
& \mathrm{A}-\mathrm{S}+\mathrm{B}-\mathrm{S} \stackrel{\boldsymbol{k}_{r}}{\longrightarrow} \mathrm{AB}_{\mathrm{g}}+2 \mathrm{~S} .
\end{aligned}
$$

This model differs from the system of the present paper in that species $B_{2}$ adsorbs dissociatively, requiring two vacant sites on the surface. Although only slightly more complex than the present system, this model exhibits rich dynamic behavior in the irreversible regime. Ziff et al. were able to construct a "phase diagram" of the steady-state coverages of both reactants as a function of the probability of adsorption of 
reactant $A, P_{A}$, where

$$
P_{A}=\frac{k_{a 1}}{k_{a 1}+k_{a 2}} .
$$

This diagram showed a jump in the steady-state coverages of both reactants from reactive to poisoned at a critical $P_{4}$ of $0.525 \pm 0.001$, indicative of instability. Presumably, with desorption of reactant, oscillations could be achieved at this point in a reversible system.

Our observations of the system size dependence of oscillatory behavior in our present model may be utilized to elucidate the role of surface defects on the oscillatory behavior of actual catalytic reactions. A major finding in this work is that thc oscillations observed are a finite size effect-they disappear on large, perfect surfaces. It is also known that oscillations of a large variety of catalytic reactions do not occur experimentally when the crystal surface is perfect, or nearly perfect, but that oscillations are found on surfaces containing defects (Yeates et al., 1985; Sault and Masel, 1982). Actual catalytic surfaces have many defects including lattice vacancies, or point defects, and slip dislocations along a particular crystallographic plane. In general, atoms are more strongly adsorbed to defects and, thus, have less affinity for reaction. It is quite conceivable that these defects can create "nucleation areas" of a sizc sufficient to produce oscillatory behavior coordinated over the entire surface. As the concentration of defects becomes less, larger regions of perfect crystal plane are available and the oscillations cease.

Our observations also indicate the significant influence of gas-phase concentration fluctuations on the dynamic behavior of catalytic systems with low rates of desorption. Previous studies of oscillatory behavior in the $\mathrm{CO}$ oxidation reaction have correlated rate of reaction oscillations with measured concentration fluctuations in an imperfectly-mixed gas inlet (Edwards et al., 1973, 1974). The "gas phase" in our simulations is implicitly well-mixed. Thus, for the conditions simulated by our algorithm, selection of a molecule to adsorb on a given site is like flipping a fair coin- $50 \%$ of the time, $A$ is chosen to adsorb and, $50 \%$ of the time, $B$ is chosen. However, even in an analagous random process of flipping a coin, there are fluctuations in the outcome scale as the square root of the number of trials (e.g. for 100 flips of a fair coin, we expect to see $50 \pm 10$ heads, on the average). In our simulations, fluctuations in the gas phase are recorded on the catalytic surface when the rate of desorption is slow. This is because reaction eliminates an equal number of As and Bs-the fluctuation is left behind on the surface. For slow rates of desorption, the fluctuation grows until poisoning of the surface occurs. However, when the rate of desorption is fast, any unreacted adsorbed species are eventually removed from the surface by desorption and the fluctuation is lost. In the irreversible limit, poisoning occurs when the number of adsorptions of one species exceeds the number of adsorptions of another by $N$, where $N$ is the total number of sites on the surface. If $N$ is the fluctuation. then approximately $N^{2}$ adsorptions are required for poisoning. Our power-law poisoning exponent of eq. (5) is presumably greater than 2 because we define time (MCS) in adsorption attempts, which may or may not be successful.

Although our findings provide a plausible explanation for the existence of oscillatory behavior on single crystal surfaces, they are insufficient to explain the synchrony of oscillations on supported catalysts or in packed-bed reactors. How do numerous and essentially isolated catalytic surfaces coordinate their oscillations to produce global kinetic oscillations? To our knowledge this remains a question which has not been adequately answered by any study completed thus far.

Monte Carlo simulations may have much to contribute to the theory of heterogeneous catalysis. In a previous paper (Fichthorn et al., 1988), we discussed the advantages of Monte Carlo and fractal scaling relationships for characterizing surface structures arising from specific catalytic mechanisms. In this paper, we have also shown that Monte Carlo simulations may enhance our understanding of the origin of chaos in catalytic reactions. The microscopic detail of a Monte Carlo simulation allows immense insight into the macroscopic behavior we see. Thus, we have been able to infer the origins of chaotic behavior in our system and to predict chaotic behavior in systems with similar features. For our simple model reaction, we found that the Langmuir-Hinshelwood mechanism with low rates of desorption is sufficient to produce the slow surface mechanism deemed necessary for selfsustained oscillations (Chang and Aluko, 1984). However, this mechanism did not produce the "perioddoubling" route to chaos observed in many dynamical systems. Rather, we observed "order through fluctuation" (Prigogine and Stengers, 1984) or deterministic chaos borne of fluctuations of increasing amplitude. In this approach to chaos, we were unable to produce the periodic or quasi-periodic oscillations observed in actual catalytic systems (Razón and Schmitz, 1986) with period-doubling. However, our model is highly simplified. Perhaps with more of the features of actual catalytic systems (e.g. adsorbate-adsorbate and adsorbate-metal interactions, heat transfer, surface diffusion), the quality of the dynamics in our model will change and match those of actual systems more closely. Since our relatively unsophisticated model already produces rich and exotic dynamics, this prospect looks quite promising. Our research will continue in this vein since it is our belief that Monte Carlo will have many future contributions to the theory of heterogeneous catąlysis.

\section{NOTATION}
A, B, chemical species

$C^{A B}, B_{2}$
$D_{f}$
$l$
correlation integral, defined by eq. (8) fractal dimension rate constant (number/time*area) length scale for correlation integral: 
either in units of the rate ( $\mathrm{rxns} / \mathrm{site} / \mathrm{MCS}$ ) or the coverage (number of sites occupied by $\mathbf{A} /$ total number of sites)

$L \quad$ linear dimension of simulation lattice $P \quad$ probability

$T_{p} \quad$ poison time for lattice in irreversible simulation (MCS)

$S \quad$ site on catalyst surface

$\mathbf{x}_{\boldsymbol{i}} \quad$ element of rate of reaction or coverage time series. For the present simulations, it is one-dimensional and is either in the units of the rate (rxns/site/MCS) or the coverage (number of sites occupied by A/total number of sites)

$\begin{array}{ll}\text { Subscripts } & \\ a & \text { adsorption } \\ d & \text { desorption } \\ \mathbf{g} & \text { gas-phase species } \\ r & \text { reaction }\end{array}$

Superscript

$z \quad$ poisoning exponent, defined by scaling in eq. (5)

\section{Greek letters}

$\xi_{i} \quad$ in eq. (9) defined by eq. (10)

$\tau$ interval between measurements in the time series of eq. (7), MCS

\section{REFERENCES}

Ben-Mizrachi, A., Procaccia, I. and Grassberger, P., 1984, Characterization of experimental (noisy) strange attractors. Phys. Rev. A 29(2), 975-977.

Chang, H. and Aluku, M., 1984, Multi-scale analysis of exotic dynamics in surface catalyzed reactions--I. Justification and preliminary model discriminations. Chem. Engng Sci. 39, 37-50.

Cox, M. P., Ertl, G., Imbihl, R. and Rustig, J., 1983, Nonequilibrium surface phase transitions during the catalytic oxidation of CO on Pt(100). Surf. Sci. 134, L517-L523.

Edwards, W. M., Worley, F. L. and Luss, D., 1973, Temperature fluctuations (flickering) of catalytic wires and gauzes--II. Experimental study of butane oxidation on platinum wires. Chem. Engng Sci. 28, 1479-1491.

Edwards, W. M., Zuniga-Chaves, J. E., Worley, F. L. and Luss, D., 1974, Causes, magnitude, and effects of temperature fluctuations (flickering) of catalytic wires and gauzes. A.I.Ch.E. I. 20, 571-581.

Eigenberger, G., 1978, Kinetic instabilities in heterogeneously catalyzed reactions -II. Oscillatory instabilities with Langmuir-type kinctics. Chem. Engng Sci. 33, $1263-1268$.

Engel, T. and Ertl, G., 1979, Elementary steps in the catalytic oxidation of carbon monoxide on platinum metals. Adv. Catal. $28.1-77$.

Fichthorn, K. A., Ziff, R. M. and Gulari, E., 1988, On the applicability of fractals and Monte Carlo methods to heterogeneous catalysis, in Catalysis 1987 (Edited by J. W. Ward), pp. 883-894. Elsevier, Amsterdam.

Fichthorn, K. A., Gulari, E. and Ziff, R. M., 1989, Noiseinduced bistability in a Monte-Carlo surface reaction model (in review)

Grassberger, P. and Procaccia, I., 1983a, Measuring the strangeness of strange attractors. Physica 9D, 189-208.

Grassberger, $I^{3}$, and Procaccia, I., 1983b, Characterization of strange attractors. Phys. Rev. Lett. 50, 346-349.

Imbihl, R., Cox, M. P., Ertl, G., Muller, H. and Brenig, W., 1985 , Kinetic oscillations in the catalytic $\mathrm{CO}$ oxidation on Pt(100): theory. J. chem. Phys. 83(4), 1578-1587.

Ivanov, E. A., Chumakov, G. A., Slinko, M. G., Bruns, D. D. and Luss, D., 1980. Isothermal sustained oscillations due to the influence of adsorbed species on the catalytic reaction rate. Chem. Engng Sci. 35, 795-803.

Jensen, K. F. and Wray, W. H., 1980, A microscopic model for catalytic surfaces-1. Catalytic wires and gauzes. Chem. Engng Sci. 35, 2439-2457.

Lindstrom, T. H. and Tsotsis, T. T., 1985, Reaction rate oscillations during $\mathrm{CO}$ oxidation over $\mathrm{Pt}-\gamma \mathrm{Al}_{2} \mathrm{O}_{3}$ : experimental observations and mechanistic causes. Surf. Sci. 150, 487-502.

Pikios, C. A. and Luss, D., 1977, Isothermal concentration oscillations on catalytic surfaces. Chem. Engng Sci. 32 $191-194$.

Prasad, S. D. and Kulkarni, B. D., 1982, Aperiodic and nearly periodic oscillations on catalytic surfaces: a simple model. Chem. Engng Sci. 37, 1117-1121.

Prigogine, I. and Stengers, I., 1984, Order out of Chaos, Chap. 6. Bantam, New York.

Rathausky, J. and Hlavacek, V., 1981, Theoretical investigation of complex dynamic behavior of the $\mathrm{CO}$ oxidation on a platinum catalyst. J. chem. Phys. 75(2), 749-756.

Razón, I. F. and Schmitz, R. A., 1986, Intrinsically unstable behavior during the oxidation of carbon monoxide on platinum. Catal. Rev. Sci. Engng 28, 89-164.

Sales, B. C., Turner, J. E. and Maple, M. B., 1982, Oscillatory oxidation of $\mathrm{CO}$ over, $\mathrm{Pt}, \mathrm{Pd}$, and Ir catalysts: theory. Surf. Sci. 114, 381-394.

Sault, A. G. and Masel, R. I., 1982, The effect of surface protrusions on self-sustained thermal oscillations during hydrogen oxidation on nickel foil. $J$. Catal. 73, 294-308.

Schwartz, S. B. and Schmidt, L. D., 1987, Is there a single mechanism of catalytic rate oscillations on Pt? Surf. Sci. 183, L269-L278.

Sheintuch, M. and Schmitz, R. A., 1977, Oscillations in catalytic reactions. Catal. Rev. Sci. Engng 15, 107-172.

Takoudis, C. G., Schmidt, L. D. and Aris, R., 1981, Steady state multiplicity in surface reactions with coverage dependent parameters. Chem. Engng Sci. 36, 1795-1802.

Wicke, F., Kummann, P., Keil, W. and Schiefler, I., 1980. Unstable and oscillatory behavior in heterogeneous catalysis. Ber. Bunsenges. phys. Chem. 84, 315-323.

Yeates, R. C., Turner, J. E., Gellman, A. J. and Somorjai, G. A., 1985, The oscillatory behavior of the $\mathrm{CO}$ oxidation reaction at atmospheric pressure over platinum single crystals: surface analysis and pressure-dependent mechanisms. Surf. Sci. 149, $175-190$.

Ziff, R. M, and Fichthorn, K. A., 1986, Fractal clustering of reactants on a catalyst surface. Phys. Rev. B 34(3), $2038-2040$.

Ziff, R. M., Gulari, F. and Barshad, Y., 1986, Kinetic phase transitions in an irreversible surface-reaction model. Phys. Rev. Lett. 5G(24), 2553-2556. 